\title{
Padé Approximation to the Solution of the Elliptical Kepler Equation
}

\author{
Aisha Alshaery* and Asrar Alsulami \\ Department of Mathematics, Faculty of Science, King Abdualaziz University, Alfaisaliah Branch, Jeddah, \\ Saudi Arabia. \\ * Corresponding author. Email: aaalshaary@kau.edu.sa \\ Manuscript submitted January 27, 2018; accepted March 5, 2018. \\ doi: 10.17706/ijapm.2019.9.1.12-20
}

\begin{abstract}
In orbital mechanics, the elliptical Kepler equation is a basic nonlinear equation which determines the eccentric anomaly of a planet orbiting the Sun. In this paper, Kepler's equation has been solved by means of Maclaurin expansion without any need to decompose the involved nonlinearity as in Adomian's method and the differential transformation method. The obtained approximate solutions are compared with a famous solution to this equation in terms of Bessel function solution. The results showed that the Maclaurin series agrees with the solution in literature in a sub domain of one revolution of the Earth around the Sun. In such a sub domain, the absolute error decreases with increasing the number of terms in the truncated series solution. In order to enhance the numerical results in the whole domain of a complete revolution, the Padé approximation for the Maclaurin series solution are established and then compared with those derived from the Bessel function solution. The comparisons for the eccentric anomaly and the approximate radial distances of the Earth from the Sun reveal that the combined Maclaurin- Padé approach is an effective tool to analyze the current problem. In addition, the domain of effectiveness exceeds the domain of a complete revolution. Moreover, the minimal distance (perihelion) and maximal distance (aphelion) approach 147 million kilometers and 152.505 million kilometers, respectively, and these results coincide with known results in astrophysics.
\end{abstract}

Key words: Kepler's equation, Padé approximation, series solution.

\section{Introduction}

In orbital mechanics, the radial distance of a planet from the Sun is given by

$$
r=a(1-e \cos \psi), 0 \leq e<1
$$

where $a$ is the semi-major axis of the ellipse, $e$ is the eccentricity of orbit, and $\psi$ is defined as the eccentric anomaly which determines the location of the planet on an auxiliary circle. The eccentric anomaly $\psi$ is used to find the corresponding true anomaly $\theta$ which determines the actual position of the planet on its elliptical path through the relationship below (see Fig. 1),

$$
\tan \left(\frac{\theta}{2}\right)=\sqrt{\frac{1+e}{1-e}} \tan \left(\frac{\psi}{2}\right)
$$

where $\psi$ is governed by Kepler's equation given as 


$$
\psi(t)-\operatorname{esin}[\psi(t)]=M=\omega t, \quad \omega=\frac{2 \pi}{T}
$$

Here, $M$ denotes to the mean anomaly and $T$ is the time of a full revolution of the planet around the Sun. To determine the true position $\theta$, Eq. (3) should be first solved for $\psi$ at a given time $t$ and then Eq. (2) is to be used. Also, the radial distance $r$ of the planet from the Sun at that instant $t$ is determined by inserting the computed value of $\psi$ into Eq. (1). Hence, the distance for any planet from the Sun is given in terms of the time $t$ provided that Kepler's equation being solved accurately for the eccentric anomaly $\psi$.

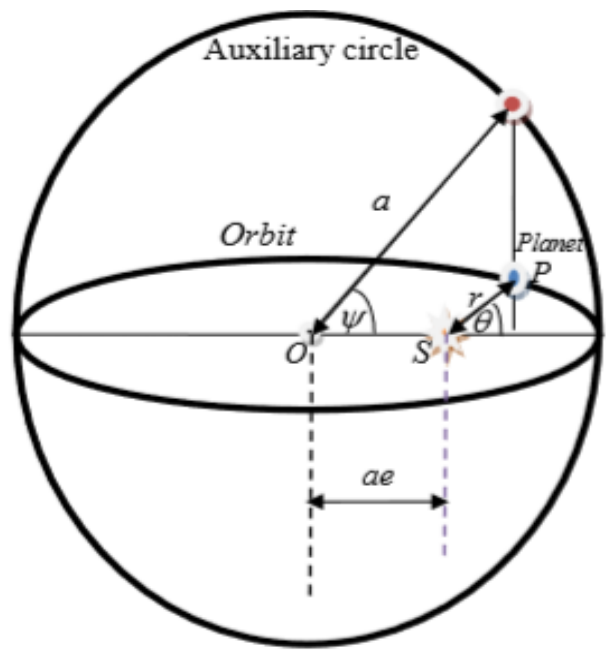

Fig. 1. The geometric relationship between the eccentric anomaly $\psi$ and the true anomaly $\theta$.

In view of Eq. (3), Kepler's equation is a nonlinear algebraic equation which has no general solution. During the past decades, a lot of papers were devoted to solving Kepler's equation for elliptic paths by using different analytical and numerical methods, see for example Refs. [1]-[6]. This equation is still of scientific interest due to its various applications in celestial mechanics.

In Ref. [1], Moulton mentioned that more than a hundred of authors have contributed on Kepler's equation, however, this list is still open (see [1] and the references therein). A fundamental but also a famous solution to Kepler's equation is discussed in Ref. [6] by Colwell who presented a mathematical proof for the solution of Eq. (3) in terms of Bessel function. Such a solution was given in the form

$$
\psi(t)=\omega t+\sum_{n=1}^{\infty} \frac{2}{n} J_{n}(n e) \sin (n \omega t)
$$

where $J_{n}$ is known as the Bessel function of order $\mathrm{n}$ defined as

$$
J_{n}(x)=\sum_{n=1}^{\infty} \frac{(-1)^{k}}{k !(k+n) !}\left(\frac{x}{2}\right)^{2 k+n}
$$

The solution given above will be taken as a reference solution for the purpose of comparison. To do that, the involved infinity should be first approximated to a finite number of terms, and this point shall be discussed later in a subsequent section.

In the past twenty years, various analytical methods have been presented to solve nonlinear algebraic equations/differential and integral equations, such as the variational iteration method, Adomian decomposition method [7]-[16], and the differential transformation method (DTM)/Taylor method [17]-[19]. It is a well-known fact that the DTM method gives the solution of nonlinear problems in the form 
an infinite series which coin cides with Taylor expansion about the zero value of the independent variable, the reason that the name of this method sometimes oscillates between the old name (DTM) and a modified name (Taylor method). However, the present authors believe that the concise title of the DTM method should be replaced by the Macluarin expansion method, even the basic rules of the DTM are applied to the investigated nonlinear problem. To make this point as clear as possible, the straightforward Macluarin expansion method shall be applied in this paper to solve Kepler's nonlinear algebraic equation without any use to rules of the DTM. To achieve this point, the equation itself shall be implemented to establish the Macluarin series solution by evaluating the successive derivatives of the unknown function when the independent variable tends to zero.

Unfortunately, for nonlinear problems in infinite domain, i.e., $[0, \infty)$, the series solution obtained by the DTM which is a Macluarin expansion is valid in a very short range of the independent variable. And in order to enlarge the domain of effectiveness, Pad'e-approximation is usually applied to the resulted series [17]-[19]. The purpose of this research is to approximate Kepler's equation by using the combined Macluarin-Pad'e method. In addition, the numerical results will be compared with those of the Bessel function solution given by Eqs. (4-5).

\section{Maclaurin Series Solution}

The Macluarin expansion is applied in this section to find a sequence of approximate solutions for Kepler's equation. The Macluarin expansion of $\psi(t)$ is defined as

$$
\begin{gathered}
\psi(t)=\sum_{k=0}^{\infty} \frac{\psi^{(k)}(0)}{k !} t^{k} \\
=\psi(0)+\psi^{(1)}(0) t+\frac{\psi^{(2)}(0)}{2 !} t^{2}++\frac{\psi^{(3)}(0)}{3 !} t^{3}+\cdots
\end{gathered}
$$

Eq. (3) is first rewritten as

$$
\psi(t)=\omega t+\operatorname{esin}[\psi(t)]
$$

On using this equation for evaluating the successive derivatives at $t=0$, noting that $\psi(0)=0$, we get

$$
\begin{gathered}
\psi^{(1)}(0)=\frac{\omega}{1-e} \\
\psi^{(3)}(0)=\frac{\omega^{3} e}{(1-e)^{4}} \\
\psi^{(5)}(0)=\frac{\omega^{5} e(1+9 e)}{(1-e)^{7}} \\
\psi^{(7)}(0)=\frac{\omega^{7} e\left(1+54 e+225 e^{2}\right)}{(1-e)^{10}} \\
\psi^{(9)}(0)=\frac{\omega^{9} e\left(1+243 e+4131 e^{2}+11025 e^{3}\right)}{(1-e)^{13}}
\end{gathered}
$$

and similarly, the rest of components can be evaluated. Accordingly, the n-terms approximate solution is given by 


$$
\Phi_{n}(t)=\sum_{k=0}^{n} \frac{\psi^{(k)}(0)}{k !} t^{k}, \quad n \geq 1
$$

Hence, the 9-terms approximate solution $\Phi_{9}(t)$ is in this form

$$
\begin{aligned}
\left(\frac{\omega}{1-e}\right) t-\left(\frac{\omega^{3} e}{3 !(1-e)^{4}}\right) t^{3}+\left(\frac{\omega^{5} e(1+9 e)}{6 !(1-e)^{7}}\right) t^{5}-\left(\frac{\omega^{7} e\left(1+54 e+225 e^{2}\right)}{7 !(1-e)^{10}}\right) t^{7}-\left(\frac{\omega^{9} e\left(1+243 e+4131 e^{2}+11025 e^{3}\right)}{9 !(1-e)^{13}}\right) t^{9} & (10)
\end{aligned}
$$

Thus, the approximate true anomaly $\theta$ and the approximate radial distance at instant $t$ are given in terms of the approximate solution $\Phi_{n}(t)$ for Kepler's equation as

$$
\theta_{n}(t)=2 \tan ^{-1}\left[\sqrt{\frac{1+e}{1-e}} \tan \left(\frac{\Phi_{n}(t)}{2}\right)\right]
$$

and

$$
r_{n}(t)=a\left[1-e \cos \left(\Phi_{n}(t)\right)\right]
$$

In view of the Bessel function solution given by Eqs. (4-5), the absolute error of the $n$-terms of Maclaurin series solution in estimating the eccentric anomaly $\psi$ is defined by

$$
E_{n}(t)=\left|\omega t+\sum_{n=1}^{\infty} \frac{2}{n} J_{n}(n e) \sin (n \omega t)-\Phi_{n}(t)\right|
$$

The expression above for the absolute error shall be used to validate the approximate solution of Maclaurin series for Kepler's equation. Furthermore, various plots for the radial distance of the Earth from the Sun shall be presented using the expression given by Eq. (12).

\section{Padé Approximations}

The $[m / n]$ Padé approximations is used to approximate a function $f(t)$ as the quotient of two polynomials of degrees $m a n d n$, respectively. It is well-known that if a function is free of singularities on the real axis, the Padé approximations usually converge on the entire real axis [20]. In order to enlarge the domain of effectiveness of the approximate solution at a wider range of the time variable, the diagonal Padé approximations are the best to achieve this goal. In this section, different diagonal Padé approximations are calculated by using MATHEMATICA 7 and presented below,

$\left[\frac{6}{6}\right](t)=\left(0.01749 t+6.13779 \times 10^{-7} t^{3}+4.51449 \times 10^{-12} t^{5}\right) /\left(1+0.00004 t^{2}+2.73686 \times\right.$ $\left.10^{-10} t^{4}-1.24158 \times 10^{-16} t^{6}\right)$

$\left[\frac{8}{8}\right](t)=\left(0.018 t+3.247 \times 10^{-7} t^{3}-2.123 \times 10^{-13} t^{5}-1.22 \times 10^{-17} t^{7}\right) /\left(1+0.00002 t^{2}-1.08 \times\right.$ $\left.10^{-11} t^{4}-8.12 \times 10^{-16} t^{6}+5.74 \times 10^{-22} t^{8}\right)$

(15)

$\left[\frac{10}{10}\right](t)=\left(0.02 t+5.5 \times 10^{-7} t^{3}+4.6 \times 10^{-12} t^{5}+8.2 \times 10^{-18} t^{7}+1.5 \times 10^{-23} t^{9}\right) /\left(1+3 \times 10^{-5} t^{2}+\right.$ $\left.2.7 \times 10^{-10} t^{4}+4 \times 10^{-16} t^{6}+5.7 \times 10^{-22} t^{8}+2.6 \times 10^{-27} t^{10}\right)$

These Padé approximations for the series solutions of Kepler's equation are obtained in the case of the orbital motion of the Earth around the Sun. The data for such orbit are fixed here, i.e., $a=150 \times 106 \mathrm{Km}$, 
$T=365.25$ days, and $e=0.0167$ [1]. Similar to Eq. (12), the absolute error of the $\left[\frac{m}{m}\right](t)$-Padé approximation is given by,

$$
E_{\left[\frac{m}{m}\right]}(t)=\left|\omega t+\sum_{n=1}^{\infty} \frac{2}{n} J_{n}(n e) \sin (n \omega t)-\left[\frac{m}{m}\right](t)\right|
$$

\section{Discussion}

Let us begin with discussing the numerical results for the expressions obtained in section 2 to approximate the solution of Kepler's equation and the radial distance of the orbital motion of the Earth around the Sun. On implementing the data for such orbit, the approximate series solutions $\Phi_{10}(t), \Phi_{15}(t)$, and $\Phi_{20}(t)$ are plotted in Fig. 2. As shown from this figure, a rapid convergence of the Maclaurin series solutions $\Phi_{10}(t), \Phi_{15}(t)$, and $\Phi_{20}(t)$ has been observed in the range $[0,228]$, i.e., less than a complete revolution of the Earth around the Sun.

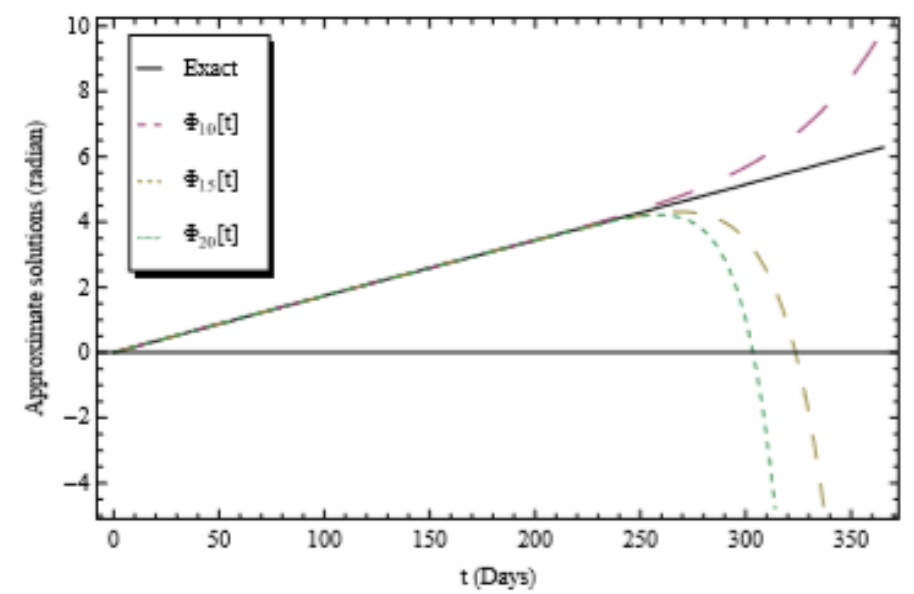

Fig. 2. The approximate solution $\Phi_{10}(t), \Phi_{15}(t)$, and $\Phi_{20}(t)$ and comparison with solution in Eq.(3).

Table 1. Calculations of the Approximate Solution $\Phi_{10}(t), \Phi_{15}(t)$, and $\Phi_{20}(t)$ and Related Absolute Errors $E_{10}(t), E_{15}(t)$, and $E_{20}(t)$ for One Revolution of Earth

\begin{tabular}{|c|c|c|c|c|c|c|c|}
\hline$t$ & $\phi_{10}(t)$ & $\phi_{15}(t)$ & $\phi_{20}(t)$ & Ref. [6] & $E_{10}(t)$ & $E_{15}(t)$ & $E_{20}(t)$ \\
\hline 0 & 0.0000000000000000 & 0.0000000000000000 & 0.0000000000000000 & 0.0000000000000000 & 0.0000000000000000 & 0.0000000000000000 & 0.0000000000000000 \\
\hline $\mathrm{T} / 8$ & 0.7973471027809634 & 0.7973471015160561 & 0.7973471015161084 & 0.7973471015161084 & $1.264854970021356 \times 10^{-9}$ & $5.23414395879653 \times 10^{-14}$ & $3.991126847115093 \times 10^{-17}$ \\
\hline $2 \mathrm{~T} / 8$ & 1.5874963605078292 & 1.5874939925554805 & 1.587493998633152 & 1.5874939987667060 & 0.000002361741123000503 & $6.211225712343914 \times 10^{-9}$ & $1.335542821988705 \times 10^{-10}$ \\
\hline $3 \mathrm{~T} / 8$ & 2.36804279483975 & 2.3678592798307427 & 2.3678639912576642 & 2.3678645642512826 & 0.0001782305884675568 & 0.000005284420539919553 & $5.72993618181568 \times 10^{-7}$ \\
\hline $4 \mathrm{~T} / 8$ & 3.145176918279056 & 3.1410021067015097 & 3.1413911067627 & 3.1415926535897930 & 0.0035842646892625565 & 0.0005905468882836341 & 0.0002015468270933788 \\
\hline $5 T / 8$ & 3.950307778096744 & 3.893557889868405 & 3.8972567397504565 & 3.9153207429283037 & 0.03498703516843966 & 0.021762853059899133 & 0.01806400317784792 \\
\hline $6 \mathrm{~T} / 8$ & 4.912918892356823 & 4.295833124172204 & 4.009758493040014 & 4.6956913084128800 & 0.21722758394394306 & 0.39985818424067676 & 0.6859328153728669 \\
\hline $7 T / 8$ & 6.477472265229439 & 0.9168404622036941 & -8.994192415833457 & 5.4858382056634780 & 0.9916340595659623 & 4.568997743459784 & 14.480030621496933 \\
\hline $8 \mathrm{~T} / 8=\mathrm{T}$ & 9.911379288631526 & -30.74845624994005 & -193.46777664414984 & 6.2831853071795860 & 3.6281939814519393 & 37.03164155711964 & 199.75096195132943 \\
\hline
\end{tabular}

In order to numerically validate these approximate solutions against Bessel function solution given by Eq. (4) where the infinity in the involved summation is approximated to fifty thousands, calculations were performed in Table 1 using MATHEMATICA 7. In addition, the approximate solutions for the radial distances 
$r_{10}(t), r_{15}(t)$ and $r_{20}(t)$ are depicted in Fig. 3. It can be concluded from Fig. 2, Table 1 and Fig. 3.

- the numerical computations of the eccentric anomaly $\psi$ using the 10-terms, 15 -terms, and the 20 -terms series solutions converge in the range $t \in[0,228]$.

- the first four columns in Table 1 show that the derived numerical results from $\Phi_{10}(t), \Phi_{15}(t)$ and $\Phi_{20}(t)$ are increasingly close to those of the Bessel function solution [6] for $t \leq \frac{5 T}{8}$.

- the last three columns show that the absolute error decreases with increasing the number of terms in the series solution for $t \leq \frac{5 T}{8}$.

- for $\mathrm{t}>\frac{5 T}{8}$, the solutions $\Phi_{10}(t), \Phi_{15}(t)$ and $\Phi_{20}(t)$ fail to give accurate solution.

- for $\mathrm{t}>\frac{5 T}{8}$, the behavior of the absolute error derived from $\Phi_{10}(t)$ is much better than the approximations $\Phi_{15}(t)$, and $\Phi_{20}(t)$. This behavior may returns to the oscillatory nature of the truncated series, since each positive term is followed by a negative term.

- the approximate solutions for the radial distances $r_{10}(t), r_{15}(t)$ and $r_{20}(t)$ converge in the range $t \in[0,228]$.

The discussion above motivates us to a further improvement, especially in the domain $t \in\left[\frac{5 T}{8}, T\right]$. In the rest of this discussion, it will be seen that the diagonal Padé approximations meet such a requirement. The diagonal Padé approximations for the eccentric anomaly $\psi$ are tabulated in Table 2 and plotted in Fig. 4. Besides, the radial distances derived from the Padé approximations are displayed in Fig. 5. It can be concluded from Fig. 4, Table 2 and Fig. 5 that

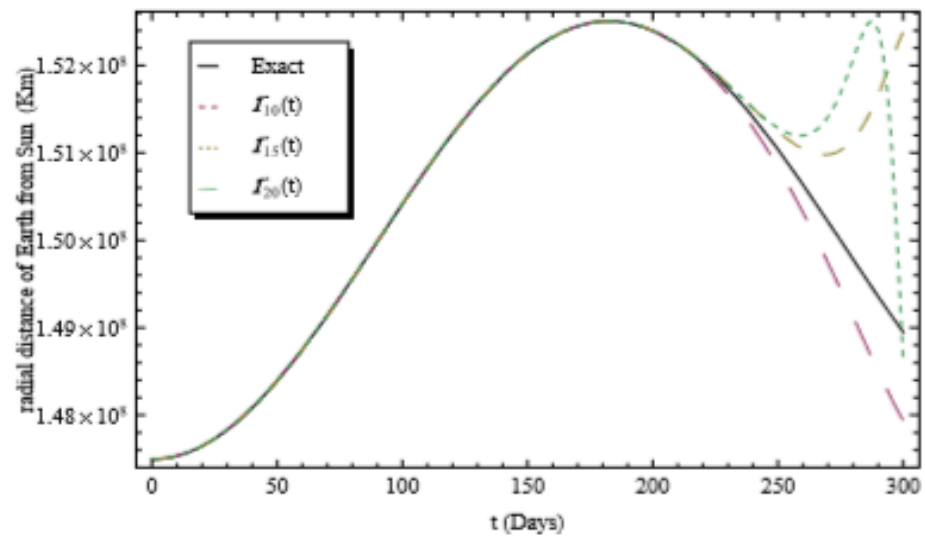

Fig. 3. The approximate solution $r_{10}(t), r_{15}(t), r_{20}(t)$ and comparison with solution in Eq.(3).

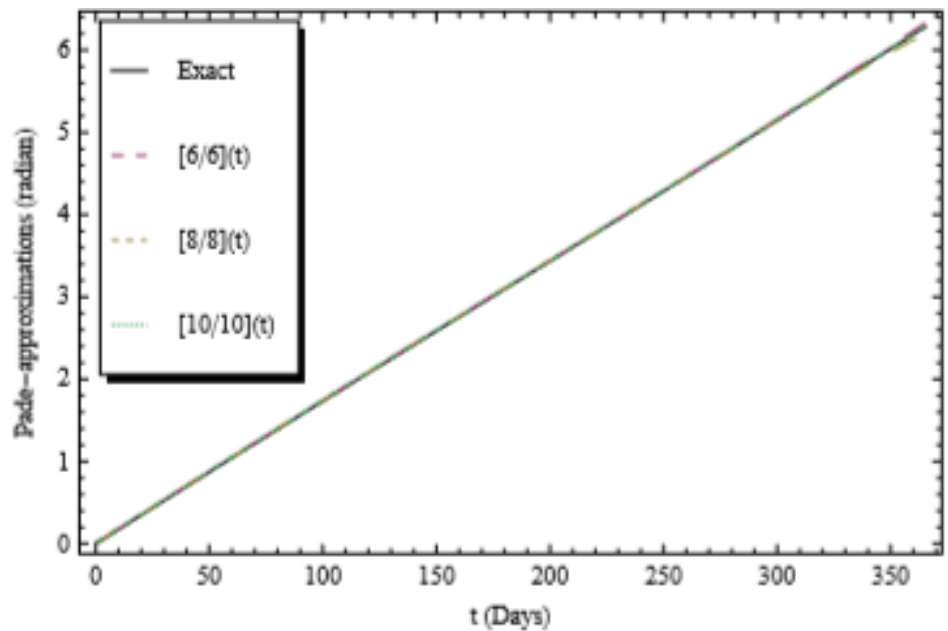

Fig. 4. The diagonal Padé approximate solutions $\left[\frac{6}{6}\right](t),\left[\frac{8}{8}\right](t)$ and $\left[\frac{10}{10}\right](t)$ and comparison with solution in Eq.(3). 


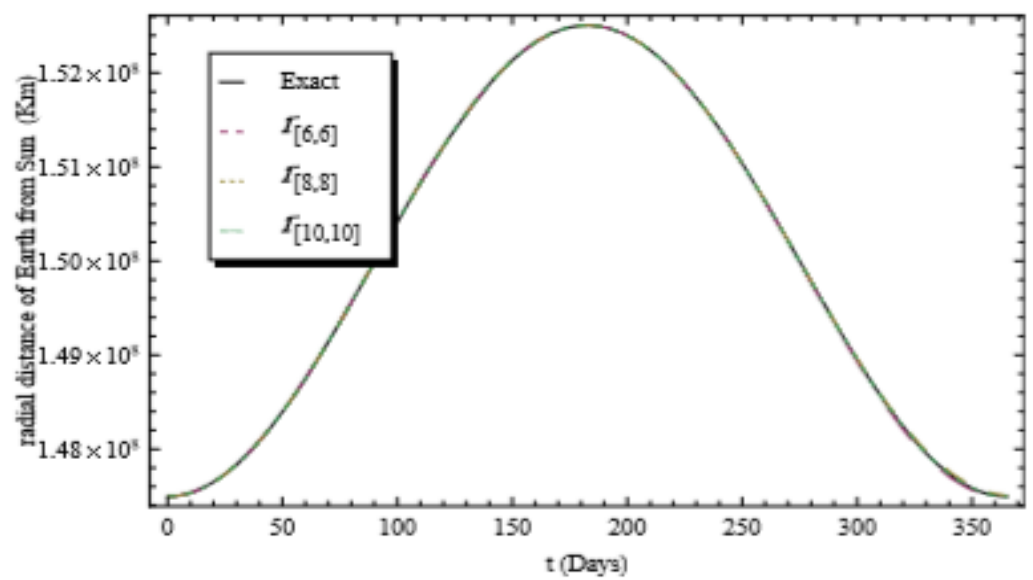

Fig. 5. The approximate radial distances $r_{\frac{6}{6}}(t), r_{\frac{8}{8}}(t)$ and $r_{\frac{10}{10}}(t)$ and comparison with solution in Eq. (3).

Table 2. Calculations of the Approximate Solution [6,6](t), [8.8] $(t)$, and $[10,10](t)$ and Related Absolute Errors $E_{[6,6]}(t), E_{[8,8]}(t)$, and $E_{[10,10]}(t)$ for One Revolution of Earth

\begin{tabular}{|c|c|c|c|c|c|c|c|}
\hline $\mathrm{t}$ & {$[6,6](t)$} & {$[8,8](t)$} & {$[10,10](t)$} & Ref. [6] & $E_{[6,6]}(t)$ & $E_{[8,8]}(t)$ & $E_{[10,10]}(t)$ \\
\hline 0 & 0.0000000000000000 & 0.0000000000000000 & 0.0000000000000000 & 0.0000000000000000 & 0.0000000000000000 & 0.0000000000000000 & 0.0000000000000000 \\
\hline$T / 8$ & 0.7973471015197036 & 0.7973471015161079 & 0.7973471015161088 & 0.7973471015161084 & $3.595095483413639 \times 10^{-12}$ & $5.83826439855434 \times 10^{-16}$ & $3.043519798446912 \times 10^{-16}$ \\
\hline $2 \mathrm{~T} / 8$ & 1.5874940202532168 & 1.587493998708935 & 1.587493998766575 & 1.587493998766706 & $2.148651077047918 \times 10^{-8}$ & $5.777114355294435 \times 10^{-11}$ & $1.310286496653249 \times 10^{-13}$ \\
\hline $3 \mathrm{~T} / 8$ & 2.3678672025105225 & 2.367864527101906 & 2.3678645638963065 & 2.3678645642512826 & 0.000002638259240451404 & $3.714937597460756 \times 10^{-8}$ & $3.549755592333752 \times 10^{-10}$ \\
\hline $4 \mathrm{~T} / 8$ & 3.141656938928234 & 3.1415896430117285 & 3.1415925822248987 & 3.141592653589793 & 0.00006428533844093718 & 0.000003010578064623694 & $7.136489444038648 \times 10^{-18}$ \\
\hline $5 \mathrm{~T} / 8$ & 3.9159694908316838 & 3.915240302437104 & 3.9153172996107957 & 3.9153207429283037 & 0.0037803415830790724 & 0.0011116622916489181 & 0.0000677340198498077 \\
\hline $6 \mathrm{~T} / 8$ & 4.699471649995959 & 4.694579646121231 & 4.69562357439303 & 4.69569130841288 & 0.0037803415830790724 & 0.0011116622916489181 & 0.0000677340198498077 \\
\hline $7 \pi / 8$ & 5.500952511229575 & 5.475461866441088 & 5.485122174802118 & 5.485838205663478 & 0.015114305566097197 & 0.010376339222389864 & 0.0007160308613595967 \\
\hline $8 T / 8=T$ & 6.328867801303486 & 6.199870490066294 & 6.2784310266403685 & 6.283185307179586 & 0.04568249412389936 & 0.08331481711329225 & 0.004754280539217777 \\
\hline
\end{tabular}

- the eccentric anomaly $\psi$ using the diagonal Padé approximations $\left[\frac{6}{6}\right](t),\left[\frac{8}{8}\right](t)$ and $\left[\frac{10}{10}\right](t)$ converge in all the domain of a complete revolution of the Earth around the Sun. Moreover, these diagonal Padé approximations coincide with the Bessel function solution [6].

- the first four columns in Table 2 show that the derived numerical results from $\left[\frac{6}{6}\right](t),\left[\frac{8}{8}\right](t)$ and $\left[\frac{10}{10}\right](t)$ are increasingly close to those of the Bessel function solution [6] for $t \in[0, T]$.

- the last three columns show that the absolute error decreases with increasing the degree of the diagonal Padé approximations.

- the last value in Table 2 indicates that the greatest absolute error obtained from $\left[\frac{10}{10}\right](t)$ is about 0.00475 in radian. The equivalent value in degrees is about 0.27 which is a very small error in estimating the eccentric anomaly $\psi$ and consequently the true anomaly of the planet Earth $\theta$.

- the approximate radial distances derived from the diagonal Pad'e approximations. i.e., $r_{\frac{6}{6}}(t), r_{\frac{8}{8}}(t)$ and $r_{\frac{10}{10}}(t)$ converge in the domain of a complete revolution of the Earth around the Sun. Furthermore, they are coincide with the approximate radial distances conducted from the Bessel function solution.

- the minimal distance of Earth from the Sun, i.e., perihelion distance, is approximately 147 million kilometers and the maximal distance, i.e., aphelion distance, approaches 152.505 million kilometers. 
It may be concluded that the present analysis for elliptical Kepler equation is simple, straightforward, and greatly coincides with the Bessel function solution.

\section{References}

[1] Moulton, F. R. (1970). An Introduction to Celestial Mechanics. Dover, New York.

[2] Ioakimids, N. I., \& Papadakis, K. E. (1985). A new simple method for the analytical solution of Kepler's equation. Celestial Mechanics and Dynamical Astronomy, 35(4), 305-316.

[3] Swerdlow, N. M. (2000). Kepler's iterative solution to Kepler's equation. Journal for History of Astronomy, 31, 339-341.

[4] Stumpf, L. (1999). Chaotic behavior in the newton iterative function associated with Kepler's equation. Celestial Mechanics and Dynamical Astronomy, 74, 95-109.

[5] Palacios, M. (2002). Kepler's equation and accelerated Newton method. Journal of Computational and Applied Mathematics, 138, 335-346.

[6] Colwell, P. (1992). Bessel functions and Kepler's equation. American Mathematical Monthly, 99(1), 45-48.

[7] Adomian, G., \& Rach, R. (1985). On the solution of algebraic equations by the decomposition method. Journal of Mathematical Analysis and Application, 105, 141-166.

[8] Adomian, G., \& Rach, R. (1986). Algebraic computation and the decomposition method. Ky-bernetes, 15(1), 33-37.

[9] El-Sayed, S. M. (2002). The modified decomposition method for solving nonlinear algebraic equations. Applied Mathematics and Computation, 132, 589-597.

[10] Babolian, E., \& J. Biazar, J. (2002). Solution of nonlinear equations by modified Adomian decomposition method. Applied Mathematics and Computation, 132, 167-172.

[11] Babolian, E., \& Javadi, S. H. (2003). Restarted Adomian method for algebraic equations. Applied Mathematics and Computation, 146(2-3), 533-541.

[12] Kaya, D., \& El-Sayed, S. M. (2004). Adomian's decomposition method applied to systems of nonlinear algebraic equations. Applied Mathematics and Computation, 154, 487-493.

[13] Awatif, A. H., \& Abulwafa, E. M. (2007). Adomian decomposition method for transient neutron transport with pomraning-eddington approximation. Proceedings of 6th International Conference on Nuclear and Particle Physics (pp. 1-16).

[14] Danish, M. (2004). Use of Adomian and restarted Adomian methods for solving algebraic equations. Journal of Basic and Applied Engineering Research, 1(10), 76-78.

[15] Adomian, G. (1994). Solving Frontier Problems of Physics: The Decomposition Method. Kluwer Academy, Boston.

[16] Wazwaz, A. M. (2005). Adomian decomposition method for a reliable treatment of the Bratu-type equations. Applied Mathematics and Computation, 166, 652-663.

[17] Ebaid, A. (2010). Approximate periodic solutions for the non-linear relativistic harmonic oscillator via differential transformation method. Communication in Nonlinear Science and Numerical Simulation, 15, 1921-1927.

[18] Ebaid, A. (2011). A reliable aftertreament for improving the differential transformation method and its application to nonlinear oscillators with fractional nonlinearities. Communication in Nonlinear Science and Numerical Simulation, 16, 528-536.

[19] Domairry, G., \& Hatami, M. (2014). Squeezing Cu-water nanofluid flow analysis between parallel plates by DTM-Pad method. Journal of Molecular Liquids, 193, 37-44.

[20] Baker, G. A.., \& Graves-Morris, P. R. (1975). Essentials of Padé Approximants. Academic, New York. 
Aisha Alshaery is an associate professor of mathematics at Faculty of Science-AL-Faisaliah Campus, King Abdulaziz University, Saudi Arabia. She obtained her PhD degree in applied mathematics from King Abdulaziz University .Her main research interest includes: analytical and numerical methods for ordinary and partial differential equations, applied mathematics and mathematical astronomy among others.

Asrar Alsulami is a student in Mathematics Department at Faculty of Science-AL-Faisaliah Campus, King Abdulaziz University, Saudi Arabia. 